\title{
CFD analysis of a twin scroll radial turbine
}

\author{
Jiří Fürst ${ }^{1, \star}$ and Zdeněk Žák ${ }^{2, \star \star}$ \\ ${ }^{1}$ Faculty of Mechanical Engineering, Czech Technical University in Prague, Karlovo nám. 13, 12135 Praha 2, Czech Republic \\ ${ }^{2}$ Centre of Vehicles for Sustainable Mobility, VTP Roztoky, Přilepská 192025263 Roztoky u Prahy, Czech Republic
}

\begin{abstract}
The contribution deals with the application of coupled implicit solver for compressible flows to CFD analysis of a twin scroll radial turbine. The solver is based on the finite volume method, convective terms are approximated using AUSM+up scheme, viscous terms use central approximation and the time evolution is achieved with lower-upper symmetric Gauss-Seidel (LU-SGS) method. The solver allows steady simulation with the so called frozen rotor approach as well as the fully unsteady solution. Both approaches are at first validated for the case of ERCOFTAC pump [1]. Then the CFD analysis of the flow through a twin scroll radial turbine and the predictions of the efficiency and turbine power is performed and the results are compared to experimental data obtained in the framework of Josef Božek - Competence Centre for Automotive Industry.
\end{abstract}

\section{Introduction}

CFD simulations play an important role in turbomachinery design and analysis. Although there are a number of ready-made software packages (both commercial and inhouse), the reliable simulations remains still a challenging task especially in the case of high rotational speed radial turbines or low-pressure stages of axial turbines. The flow passing through such types of turbines achieves high Mach number and has to therefore considered as compressible.

Most software packages for the simulation of compressible flows are based on finite volume approach and use either a segregated or a coupled approach. When using the segregated solver the equations expressing conservation of momentum, density, and total energy are solved independently in a loop in order to obtain steady state or unsteady solution. The algorithm is usually based on the so called SIMPLE algorithm proposed originally Patankar and Spalding in [2] for stationary case, or on the PISO algorithm of Issa [3] for unsteady case. Although the segregated solvers have been extended also to compressible flow problems including transonic or supersonic flows (see e.g. [4] or [5]), the efficiency of these method is in the case of high speed flows usually worse than the efficiency of implicit fully coupled solvers [6].

So far several attempts have been done in order to develop coupled solvers for OpenFOAM platform. Let us mention the solver based on the central-upwind scheme of Kurganov and Tadmor [7] or the density based solvers on OpenFOAM-extend package based on AUSM+up [8] or HLLC [9] fluxes. Unfortunately all these solvers use explicit time-marching method which puts a strong limit to the time step and therefore the efficiency is very weak

\footnotetext{
${ }^{\star}$ e-mail: Jiri.Furst@fs.cvut.cz

$\star \star$ e-mail: Zdenek.Zak@fs.cvut.cz
}

especially for steady state flows at high Reynolds number. Therefore we developed in [6] an implicit coupled solver based on the symmetric lower-upper Gauss-Seidel procedure, see e.g. [10] or [11] and we further extend this solver for the case of turbomachinery flows using either the frozen rotor or unsteady approaches.

\section{LU-SGS solver for turbomachinery flows}

\subsection{Description of the solver}

The system of time averaged Navier-Stokes equations in conservative form are given as follows

$$
\begin{array}{r}
\frac{\partial \rho}{\partial t}+\nabla \cdot(\rho \vec{V})=0, \\
\frac{\partial(\rho \vec{U})}{\partial t}+\nabla \cdot(\rho \vec{V} \otimes \vec{U}-\vec{\tau})+\nabla p+\rho \vec{\Omega} \times \vec{U}=0, \\
\frac{\partial(\rho E)}{\partial t}+\nabla \cdot(\rho E \vec{V}+p \vec{U}-\vec{\tau} \cdot \vec{U}-k \nabla T)=0,
\end{array}
$$

where $\rho$ is the density, $\vec{U}$ is the absolute velocity vector, $\vec{V}=\vec{U}-\vec{\Omega} \times \vec{x}$ is the velocity vector in the reference frame rotating with constant angular velocity $\vec{\Omega}, p$ is the pressure, $\vec{\tau}$ is the (effective) stress tensor, $E$ is the specific total energy, $k$ is the (effective) thermal conductivity, and $T$ is the temperature. The system is closed by the equation of state for ideal gas $p / \rho=r T$.

The discretization in space is achieved using finite volume method where convective fluxes are replaced by an approximate Riemann solver (in our case with the HLLC flux, see [9]) and viscous fluxes are discretized using central differences. The temporal discretization is achieved by using the implicit Euler method of first or second order, thus for the first order method

$$
\vec{W}_{i}^{n+1}=\vec{W}_{i}^{n}-\frac{\Delta t}{\left|\Omega_{i}\right|} \vec{R}\left(\vec{W}^{n+1}\right)_{i} .
$$


Here $\vec{W}=[\rho, \rho \vec{u}, \rho E]$ and $\vec{R}(\vec{W})$ is the discretization of spatial terms.

The resulting system of non-linear equations is then solved by a matrix-free lower-upper symmetric GaussSeidel method using the following two step procedure:

$$
\begin{aligned}
\boldsymbol{D}_{i} \Delta \vec{W}_{i}^{(1)} & =-\vec{R}_{i}-\frac{1}{2} \sum_{j<i}\left[\Delta \boldsymbol{F}_{j}^{(1)} \cdot \vec{S}_{i j}+\lambda_{i j}^{*} \Delta \vec{W}_{j}^{(1)}\right], \\
\boldsymbol{D}_{i} \Delta \vec{W}_{i} & =\boldsymbol{D}_{i} \Delta \vec{W}_{i}^{(1)}-\frac{1}{2} \sum_{j>i}\left[\Delta \boldsymbol{F}_{j} \cdot \vec{S}_{i j}+\lambda_{i j}^{*} \Delta \vec{W}_{j}\right] .
\end{aligned}
$$

Here

$$
\begin{aligned}
\Delta \vec{W}_{i}^{(1)} & =\vec{W}_{i}^{(1)}-\vec{W}_{i}^{n}, \\
\Delta \boldsymbol{F}_{i}^{(1)} & =\boldsymbol{F}\left(\vec{W}_{i}^{(1)}\right)-\boldsymbol{F}\left(\vec{W}_{i}^{n}\right), \\
\Delta \boldsymbol{F}_{i} & =\boldsymbol{F}\left(\vec{W}_{i}^{n+1}\right)-\boldsymbol{F}\left(\vec{W}_{i}^{n}\right),
\end{aligned}
$$

$\lambda^{*}$ being the spectral radius of jacobains of fluxes and

$$
\boldsymbol{D}_{i}=\left(\frac{\left|\Omega_{i}\right|}{\Delta t_{i}}+\frac{1}{2} \sum_{j \in N_{i}} \lambda_{i j}^{*}\right) \boldsymbol{I} .
$$

See [10] or [6] for details.

In order to solve turbomachinery flows the solver has been extended to support calculations both in multiple reference frames allowing the so called frozen-rotor method and using the so called sliding mesh method [12].

\subsection{Validation for the case of ERCOFTAC pump}

In order to validate the solver and to assess suitability of frozen rotor approach a simulation of flow through a twodimensional model of the ERCOFTAC radial pump has been performed. The results are compared to experimental data obtained by Ubaldi et al. [1]. The case was previously studied by [13] assuming incompressible flow and detailed data together with numerical results were published in the form of validation test case for evaluation of rotor-stator interaction.

The simplified model of the pump has 7 impeller blades, 12 diffuser vanes and $6 \%$ vaneless radial gap. The parameters of the model are given bellow:

\section{Impeller :}

- Inlet blade diameter $D_{1}=240 \mathrm{~mm}$,

- Outlet blade diameter $D_{2}=420 \mathrm{~mm}$,

- Number of blades $Z_{i}=7$,

- Blade span $z=40.4 \mathrm{~mm}$,

\section{Diffuser :}

- Inlet vane diameter $D_{3}=444 \mathrm{~mm}$,

- Outlet vane diameter $D_{2}=664 \mathrm{~mm}$,

- Number of vanes $Z_{d}=12$,

Operating conditions :

- Rotational speed $n=2000 \mathrm{rpm}$,

- Impeller tip speed $U_{2}=43.98 \mathrm{~m} / \mathrm{s}$,

- Flow rate coefficient $\phi=4 Q /\left(U_{2} \pi D_{2}^{2}\right)=0.048$,

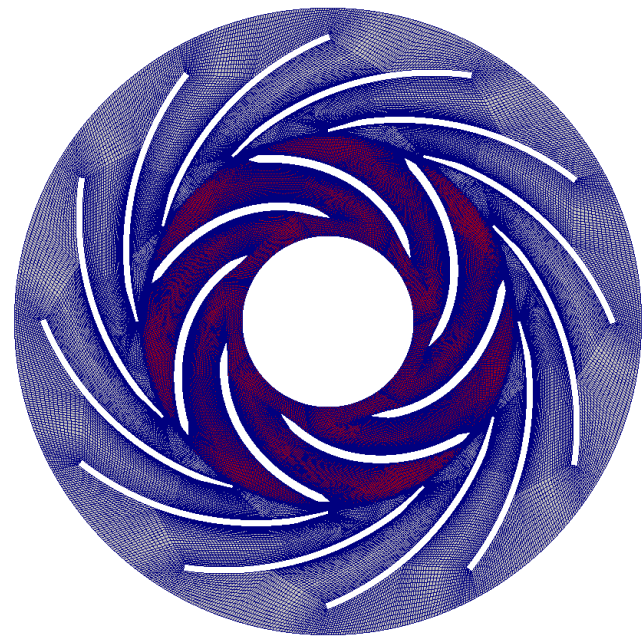

Fig. 1. Structured 2D multiblock mesh for ERCOFTAC centrifugal pump, rotating zone in red.

- Flow rate $Q=0.292 \mathrm{~m}^{3} / \mathrm{s}$,

- Reynolds number $R e=\frac{U_{2} D_{2}}{2 v}=6.5 \times 10^{5}$.

The numerical solution has been calculated using a domain with inlet diameter $D_{0}=200 \mathrm{~mm}$ and outlet diameter $D_{4}=730 \mathrm{~mm}$. The multi-block structured mesh provided by Petit at al. [13] contains 93886 quadrilateral cells with average near wall cell size about $y_{1}^{+} \approx 20$, see fig. 1 .

\subsubsection{Steady state frozen rotor simulation}

In order to assess the accuracy and suitability of frozen rotor approach two simulations have been run. First one using standard segregated solver based on SIMPLE loop (namely simpleFoam) assuming incompressible flow and the second one using coupled LU-SGS scheme for compressible flow.

Left half of figure 2 shows the absolute velocity magnitude obtained with segregated solver and the right half shows results obtained with coupled LU-SGS solver. One can see that both models give very similar flow field.

Figures 3 and 4 show a comparison of the radial and tangential components of the velocity at impeller outlet $D_{m} / D_{2}=1.02$ obtained with segregated and LU-SGS solver with experimental data of [1]. Note that the velocity is normalized by the impeller tip speed and the arc length $y_{i}$ is normalized by the impeller pitch $G_{i}=\pi D_{2} / Z_{i}$. These results correspond to data obtained with another numerical method based on segregated approach by Petit et al. [14].

One can see that the frozen approach is capable to resolve some basic features of the flow. Namely the radial component of the velocity corresponds well to experimental data. The tangential velocity is slightly over-estimated by both calculations. On the other hand the shape and position of wakes is completely wrong due to frozen rotor approach. 


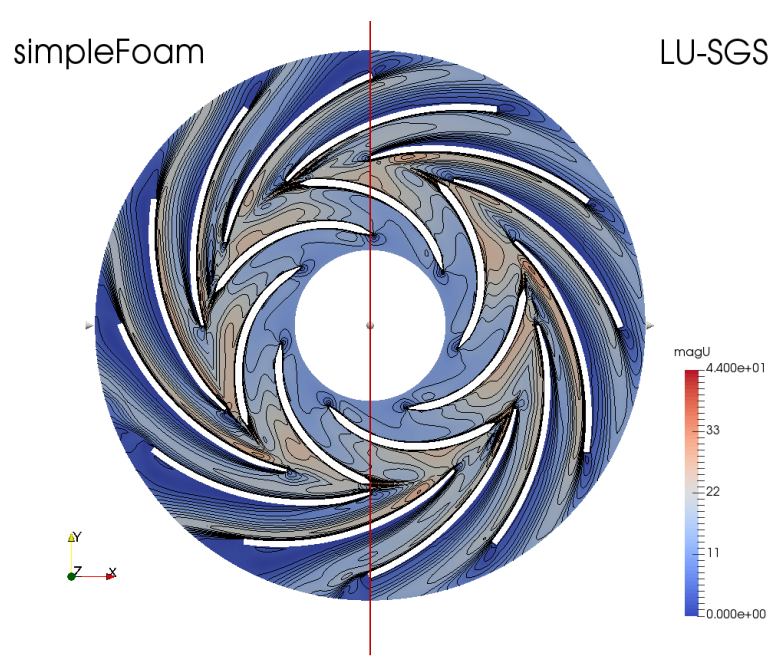

Fig. 2. Comparison of calculated velocity magnitude using frozen rotor approach and segregated solver (left part) or coupled LU-SGS solver (right part).

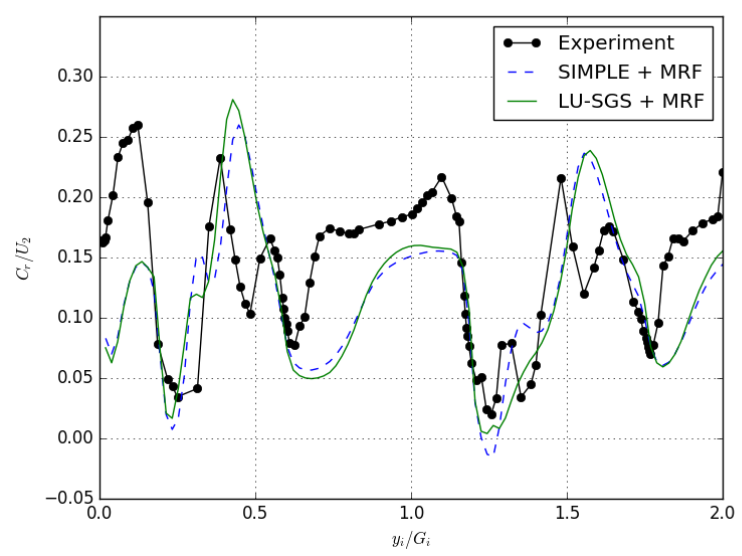

Fig. 3. Radial component of absolute velocity at the ERCOFTAC impeller outlet, frozen rotor approach.

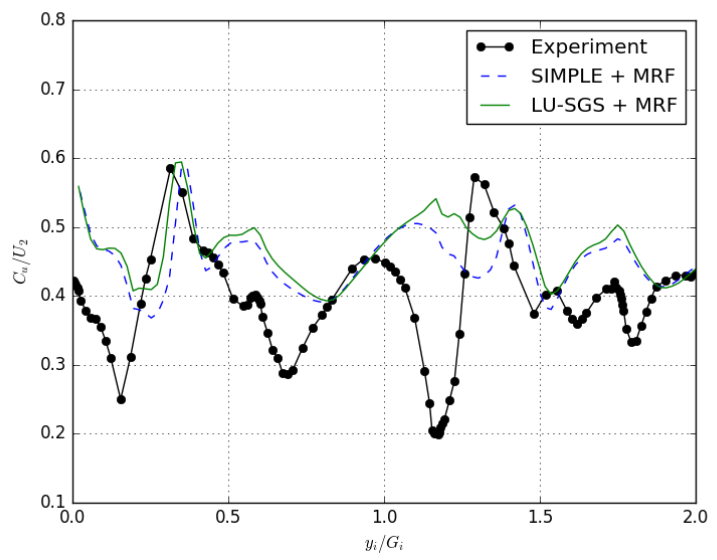

Fig. 4. Tangential component of absolute velocity at the ERCOFTAC impeller outlet, frozen rotor approach.
Table 1. Efficiency evaluation of the ERCOFTAC pump.

\begin{tabular}{|c|c|c|c|c|}
\hline & $Q\left(\mathrm{~m}^{3} / \mathrm{s}\right)$ & $\Delta p(\mathrm{~Pa})$ & $M(\mathrm{Nm})$ & $\eta(\%)$ \\
\hline \multicolumn{5}{|c|}{ Steady state simulation } \\
\hline LU-SGS & 0.296 & 931.12 & 1.48 & 88.9 \\
simpleFoam & 0.292 & 921.21 & 1.43 & 89.6 \\
\hline \multicolumn{5}{|c|}{ Unsteady simulation } \\
\hline LU-SGS & 0.296 & 940.90 & 1.43 & 93.1 \\
pimpleFoam & 0.292 & 931.09 & 1.39 & 93.6 \\
\hline
\end{tabular}

\subsubsection{Unsteady sliding mesh simulation}

In order to resolve unsteady rotor-stator interactions the unsteady simulation with moving rotor zone had to be carried out. The calculation has been run with the same mesh as in the previous case using second order backward differencing in time combined with dual time stepping technique in the case of LU-SGS solver. The physical time step was set to $\Delta t=3 \times 10^{-5} \mathrm{~s}$ which corresponds to $1 / 1000$ of revolution period.

Figure 5 shows comparison of phase locked ensemble averaged velocity components calculated with coupled LU-SGS solver with results obtained with segregated solver (pimpleDyMFoam) and with experimental data of Ubaldi et al. [1]. The figures correspond to dimensionless time $t / T_{i}=0.126,0.226,0.326$, and 0.426 where $T_{i}=2 \pi /(\omega Z i)$ is the impeller blade passing time.

One can see that both solvers predict very similar velocity at the impeller outlet. The most pronounced difference is in the tangential component of the velocity for $t / T i=0.126$, see fig. 5b. The coupled solver predicts higher peak in the velocity component at $y_{i} / G_{i} \approx 0.3$ whereas the results obtained with segregated solver correspond very well to experimental data.

The table 1 sumarizes results obtained with coupled LU-SGS solver for compressible flow and with segregated solver for incompressible flows. One can see that the LUSGS solver predicts both in steady and unsteady cases slightly higher pressure rise $\Delta p$ and torque $M$. On the other hand both solvers predict similar pump efficiency $\eta=\Delta p Q /(M \omega)$. The efficiency predicted by steady-state frozen rotor simulations is slightly lower than the one calculated with unsteady method, nevertheless the difference isn't excessively large and therefore the frozen rotor approach is a viable method for estimating efficiency of the pump.

\section{Analysis of twin scroll turbine}

Twin scroll radial centripetal turbocharger turbines are used in the automotive industry. The twin scroll design is beneficial in conjunction with the internal combustion 


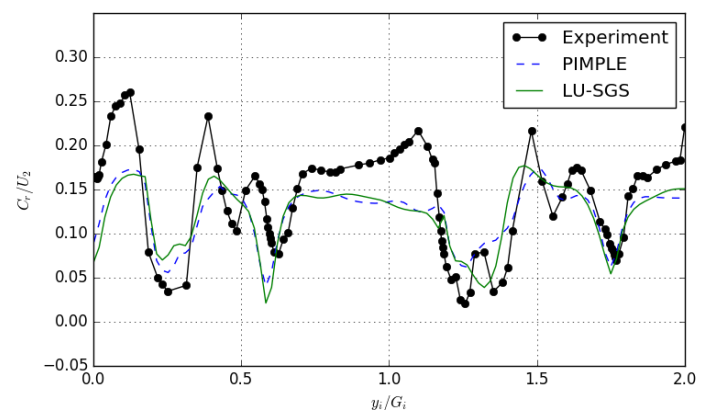

(a) Radial velocity, $t / T_{i}=0.126$.

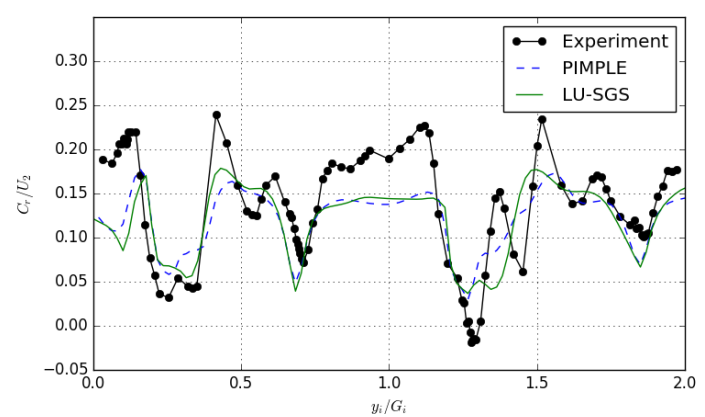

(c) Radial velocity, $t / T_{i}=0.226$.

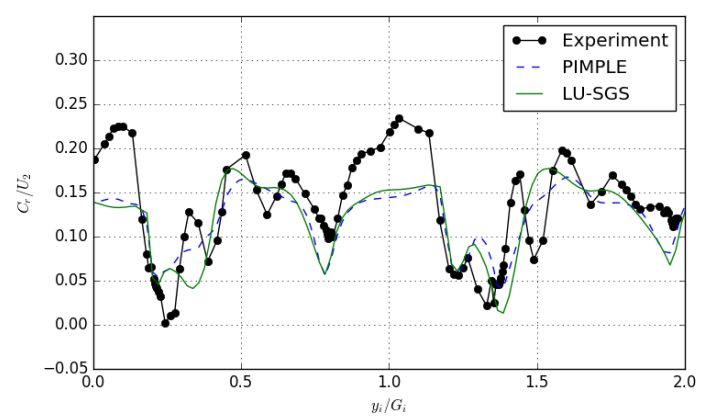

(e) Radial velocity, $t / T_{i}=0.326$.

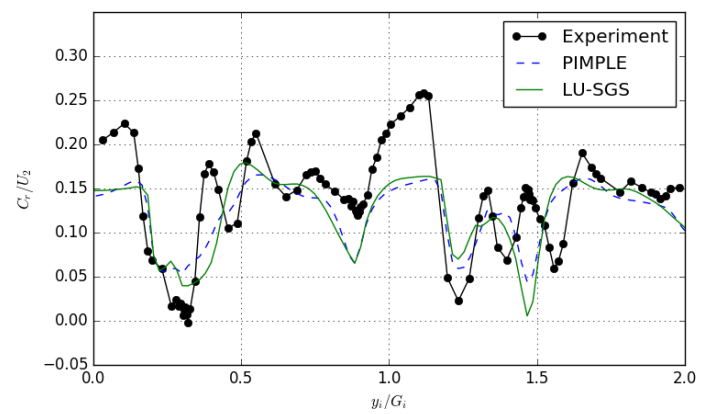

(g) Radial velocity, $t / T_{i}=0.426$.

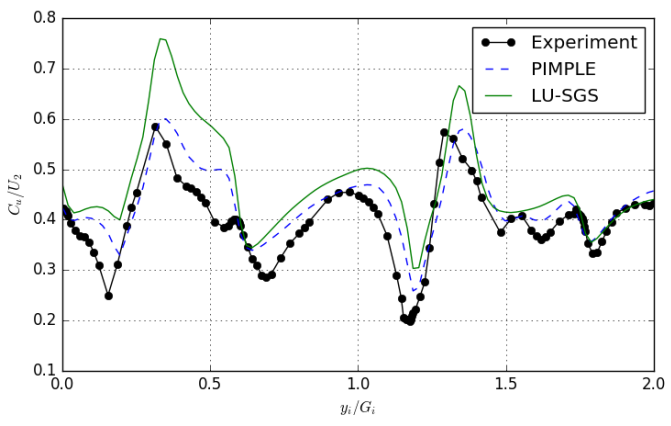

(b) Tangential velocity, $t / T_{i}=0.126$.

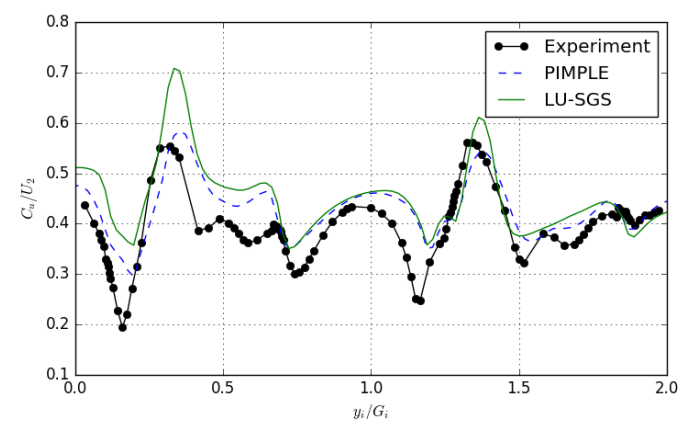

(d) Tangential velocity, $t / T_{i}=0.226$.

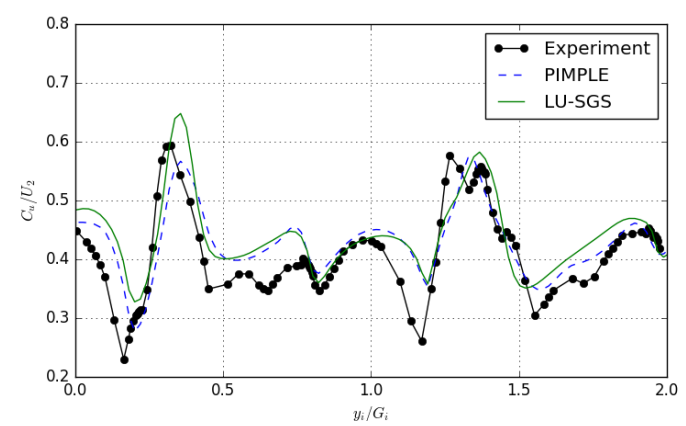

(f) Tangential velocity, $t / T_{i}=0.326$.

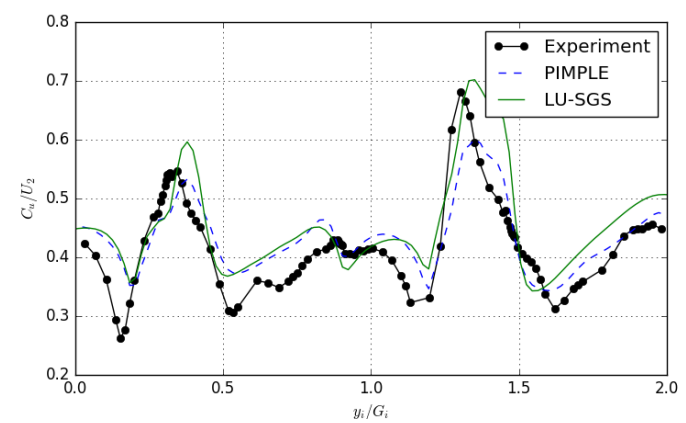

(h) Tangential velocity, $t / T_{i}=0.426$.

Fig. 5. Ensemble averaged velocity components at the ERCOFTAC impeller outlet. 


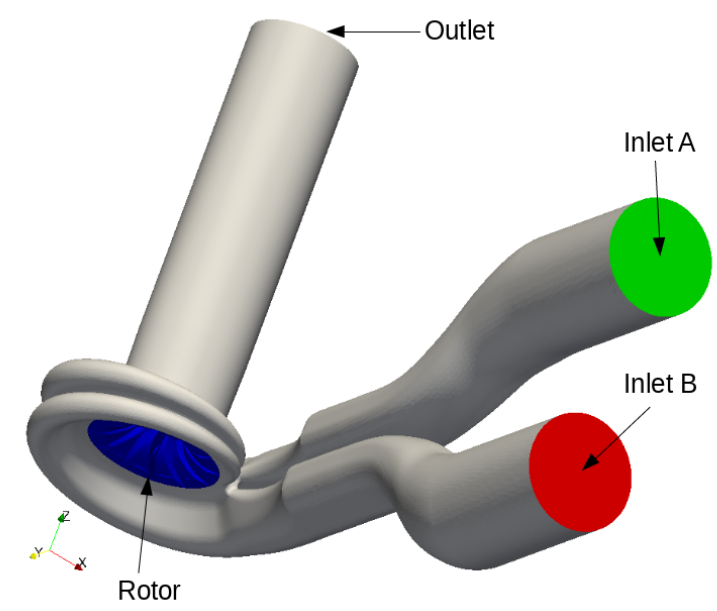

Fig. 6. Computational domain for twin scroll turbine simulations.

engine with firing interval between cylinders around 240 degrees in exhaust manifold branches, i.e. six cylinder engines. The advantages consist in the higher efficiency of a turbine and better engine response during the transients due to better utilization of exhaust gas energy. The twin scroll turbines are also used for the four cylinder engines to improve the engine behaviour during transients. The firing interval between cylinders in mentioned case is $360^{\circ}$ in exhaust manifold branches, thus out of optimum and the high pulsating flow upstream of a turbine cannot be utilized as effective as in the case of six cylinder engine.

The tested twin scroll turbine with symmetric design of sections is used for turbocharging of a six cylinder Diesel engine. The impeller has 12 vanes. The impeller diameter at inlet equals to $76.6 \mathrm{~mm}$ and averaged outlet diameter equals to $46.7 \mathrm{~mm}$.

\subsection{Simulation setup}

Due to high rotational speed together with high expansion ratio, the maximal Mach number exceeds one in some cases and the flow has to be definitely considered as a compressible gas. Simulations have been run with LUSGS solver which is developed especially for dealing with transonic flows. The domain (see figure 6) was divided to three zones: the stationary inlet zone containing both inlet tubes and volutes, the rotating zone containing turbine rotor, and the stationary zone including outlet tube. Each zone was meshed independently using snappyHexMesh software and the three parts were then glued into single mesh with interfaces between zones. The final unstructured hexa-dominant mesh contained $2.2 \times 10^{6}$ cells with near-wall mesh size $y_{1}^{+}<160$, (60 at the rotor) with average $y_{1}^{+} \approx 10$, see fig. 7 and 8 ..

The spatial discretization was performed using second order scheme with Barth-Jaspersen limiter [15] and HLLC flux [9] for convective terms and central scheme for diffusive terms. The implicit Euler method with local time stepping was used for advancing in pseudo-time in the case of

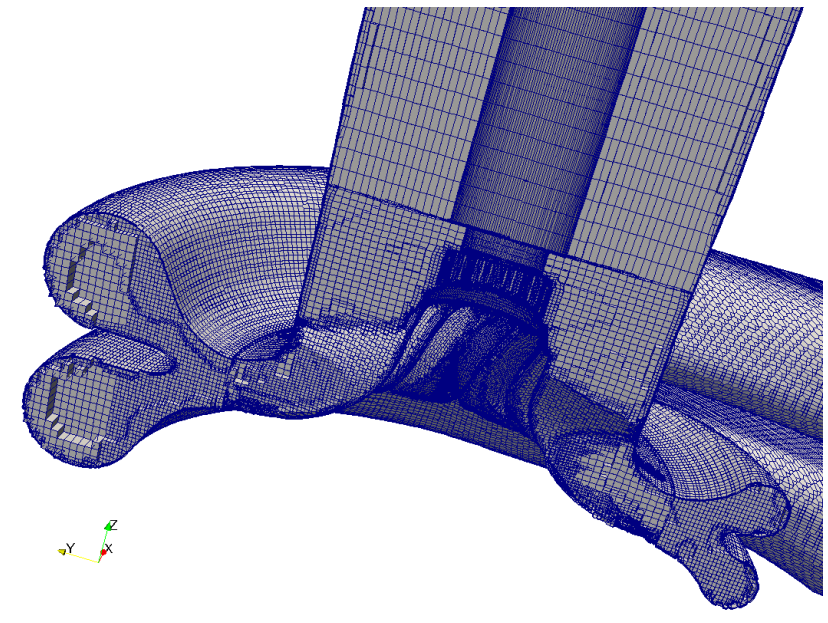

Fig. 7. Computational mesh for twin scroll turbine simulations.

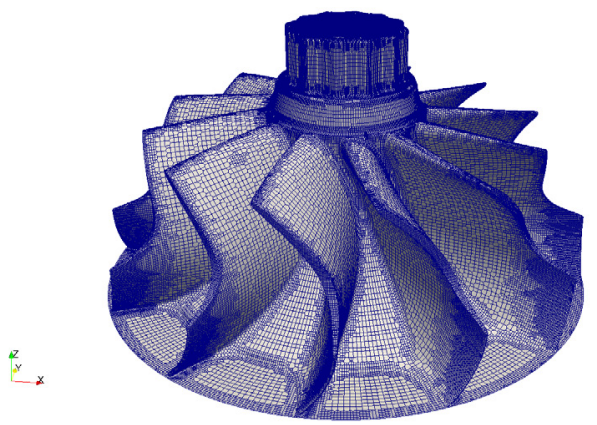

Fig. 8. Surface mesh at the rotor for twin scroll turbine simulations.

steady frozen rotor simulation whereas second order backward Euler method combined with local pseudo-time stepping was used in the transient case.

The flow regime was characterized by the following boundary conditions:

- Inlets - the value of total pressures $p_{0 A}, p_{0 B}$ and total temperatures $T_{0 A}, T_{0 B}$ was prescribed at both inlets. The velocity direction was normal to the inlet planes and the inlet turbulence intensity $T u=5 \%$ together with mixing length scale $l=2.8 \mathrm{~mm}$ was assumed in all cases.

- Outlet - the value of static pressure $p_{\text {out }}$ was prescribed according to given expansion ratio.

- Shroud - the no-slip condition was assumed together with $\partial p / \partial n$ and given wall temperature $T_{\text {wall }}$ at the shroud. Due to relatively coarse mesh the wall functions were used for turbulence model.

- Rotor - the same set of boundary conditions as for the shroud was assumed here with the exception of velocity at the rotor wall which was set to the velocity of the rotor.

The gas was governed by the ideal equation of state with constant heat capacity $c_{p}$ corresponding to air at the 


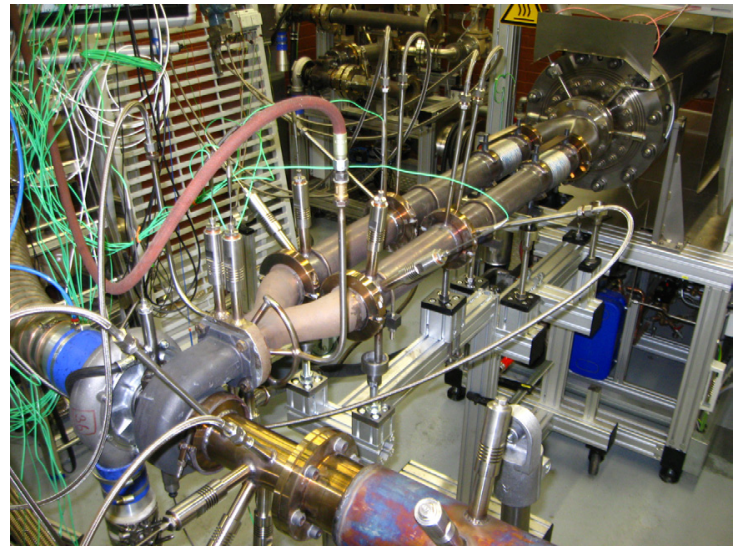

Fig. 9. Twin scroll turbocharger test bed with separated sections upstream of a turbine
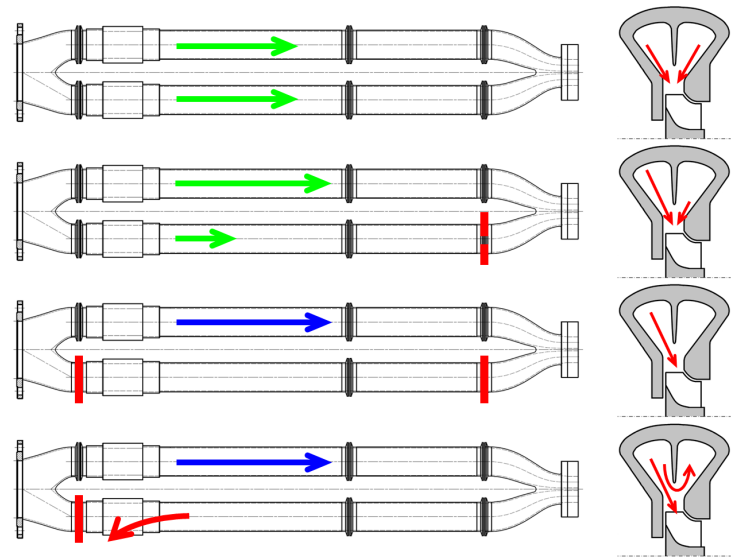

Fig. 10. Turbocharger test bed (down from top) - uniform admission; partial admission (throttling in one turbine section); extreme partial admission with closed section; backflow

temperature $T_{\text {wall }}$. The effects of turbulence were modeled by Menter's SST turbulence model [16].

The table 2 sumarizes boundary conditions and gas properties used for the simulations.

\subsection{Experimental data}

The goal of the experiments on the steady flow test bed (see fig. 9), with separated sections upstream of a turbine, was to obtain a sufficient set of data, which describes the twin scroll turbine behaviour under different level of the impeller admission. The specific hot gas stand with open loop is suitable for achievement of the arbitrary level of turbine impeller admission. The unequal partial level of admission is reached via the throttling in a turbine section and extreme partial admission by closure of one section, see the sketch at fig. 10 .

The selected twin scroll turbine was tested at different pressure ratios, relatively broad range of load and under different levels of the impeller admission. The turbine load is described by the blade speed ratio - BSR. Low blade speed ratios, i.e. high load of the turbine, were achieved by the combination of the compressor with larger wheel as a load and relatively high temperature upstream of the turbine about $1100 \mathrm{~K}$. With the temperature of exhaust gases about $900 \mathrm{~K}$ upstream of the turbine, the turbine was able to run with isentropic efficiency close to optimum. The turbine, driven by cold air only, was unloaded and achieved high blade speed ratios. Three levels of impeller admission were measured in our case - full, partial (throttling in one section) and extreme partial with closed section.

\subsection{Results}

Although the experimental data covers different partial levels of admission, the CFD study was limited to cases with uniform admission only. The results of simulations were postprocessed in order to make comparison with experimental data. First, the reduced mass flow rate defined by

$$
\dot{m}_{\text {red }}=\left(\dot{m}_{A}+\dot{m}_{B}\right) \frac{\sqrt{T_{0 A B}}}{p_{0 A B}} 10^{5},
$$

was evaluated. Here $\dot{m}_{A}$ and $\dot{m}_{B}$ are the mass flow rates through inlet $\mathrm{A}$ and $\mathrm{B}, T_{0 A B}=\left(T_{0 A}+T_{0 B}\right) / 2$, and $p_{0 A B}=$ $\left(p_{0 A}+p_{0 B}\right) / 2$. Next, the torque $M$ has been evaluated and used for the rotor power calculation

$$
P_{\text {rotor }}=M \omega_{\text {rotor }}
$$

The ventilation losses were estimated using empirical formula

$$
P_{\text {vent }}=K \rho_{2} D_{2}^{2} U_{2}^{3} R e_{2}^{-0.2}
$$

where $K=0.07$ is an empirical constant, $D_{2}$ is the rotor diameter, $U_{2}=\omega_{\text {rotor }} D_{2} / 2$ is the rotor inlet edge speed and $R e_{2}=\rho_{2} D_{2} U_{2} /$ is the Reynolds number and finally the turbine efficiency was evaluated as

$$
\eta=\frac{P_{\text {rotor }}-P_{v e n t}}{\left(\dot{m}_{A}+\dot{m}_{B}\right) c_{p} T_{0 A B}\left(1-\left(\frac{p_{\text {out }}}{p_{0 A B}}\right)^{\frac{\kappa-1}{\kappa}}\right)} \cdot 100 \% .
$$

Note that in the case of transient simulations calculations have been started from steady the initial condition corresponding to steady state frozen rotor simulation and the ten revolutions of the rotor have been calculated. The resulting mass flow rate and torque were then calculated by averaging over the last revolution. Figure 11 shows a time dependent torque obtained by transient simulation for TP1 regime. From the amplitude spectrum displayed at the figure 12 one can see that time dependent signal contains only one important harmonics corresponding to the blade passage frequency. Similar results were obtained also for other regimes.

Figures 13, 14, and 15 display the distribution of the pressure at solid and rotating walls, the absolute Mach number and velocity vectors in the longitudinal cut through the domain, and the relative Mach number in the rotor zone for TP1 regime. One can see that both absolute and relative Mach numbers exceed unity and therefore the compressibility plays an important role in the simulations.

The table 4 shows the comparison of experimental data with the results of steady and unsteady simulations. 
Table 2. Boundary conditions and gas properties for twin scroll turbine analysis

\begin{tabular}{|l|r|r|r|r|r|r|r|r|r|}
\hline Run & $p_{0 A}(\mathrm{~Pa})$ & $p_{0 B}(\mathrm{~Pa})$ & $p_{\text {out }}(\mathrm{Pa})$ & $T_{0 A}(\mathrm{~K})$ & $T_{0 B}(\mathrm{~K})$ & $T_{\text {wall }}(\mathrm{K})$ & $R P M(\mathrm{rpm})$ & $c_{p}(\mathrm{~J} / \mathrm{kg} / \mathrm{K})$ & $\kappa(-)$ \\
\hline \hline TP 1 & $2.070 \times 10^{5}$ & $2.077 \times 10^{5}$ & $9.232 \times 10^{4}$ & 877.030 & 891.656 & 800 & 104850 & 1097.997 & 1.354 \\
\hline TP 2 & $2.066 \times 10^{5}$ & $2.075 \times 10^{5}$ & $9.236 \times 10^{4}$ & 874.636 & 889.859 & 800 & 109011 & 1097.997 & 1.354 \\
\hline TP 3 & $2.084 \times 10^{5}$ & $2.092 \times 10^{5}$ & $9.146 \times 10^{4}$ & 1077.040 & 1080.259 & 1000 & 88776 & 1142.580 & 1.336 \\
\hline TP 4 & $1.544 \times 10^{5}$ & $1.550 \times 10^{5}$ & $9.512 \times 10^{4}$ & 873.127 & 891.125 & 800 & 85227 & 1097.997 & 1.354 \\
\hline TP 5 & $2.063 \times 10^{5}$ & $2.067 \times 10^{5}$ & $9.333 \times 10^{4}$ & 288.478 & 288.191 & 288 & 73521 & 1005.051 & 1.400 \\
\hline TP 6 & $1.545 \times 10^{5}$ & $1.550 \times 10^{5}$ & $9.296 \times 10^{4}$ & 1075.105 & 1080.552 & 1000 & 72814 & 1142.580 & 1.336 \\
\hline TP 7 & $1.550 \times 10^{5}$ & $1.554 \times 10^{5}$ & $9.310 \times 10^{4}$ & 1079.464 & 1084.990 & 1000 & 67489 & 1142.580 & 1.336 \\
\hline TP 8 & $1.216 \times 10^{5}$ & $1.219 \times 10^{5}$ & $9.654 \times 10^{4}$ & 881.779 & 888.423 & 800 & 63430 & 1097.997 & 1.354 \\
\hline TP 9 & $1.534 \times 10^{5}$ & $1.535 \times 10^{5}$ & $9.623 \times 10^{4}$ & 289.580 & 289.341 & 288 & 57048 & 1005.051 & 1.400 \\
\hline TP 10 & $1.219 \times 10^{5}$ & $1.223 \times 10^{5}$ & $9.629 \times 10^{4}$ & 874.801 & 888.021 & 800 & 54418 & 1097.997 & 1.354 \\
\hline
\end{tabular}

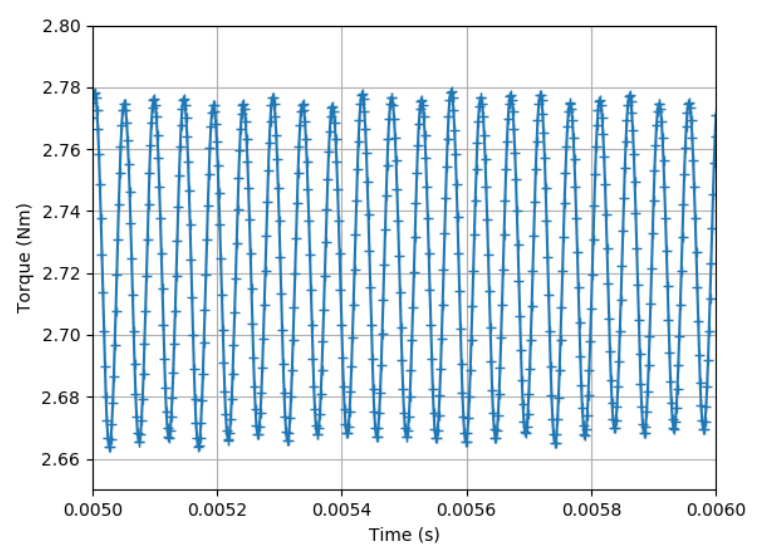

Fig. 11. Time dependent torque as a result of transient simulation for TP1 regime.

Figures 16 and 17 show graphically the reduced mass flow rates and turbine efficiency as a function of turbine expansion ratio $\Pi=p_{0 A B} / p_{\text {out }}$. Each number represents corresponding regime (see table 2) and its color corresponds to a method for obtaining the result: black color means experimental data, red color means steady state $\mathrm{CFD}$, and blue color represents transient CFD. Both figures contains also lines displaying results of steady state calculation with three fixed rotation speeds $50000 \mathrm{rpm}$, $75000 \mathrm{rpm}$ and $100000 \mathrm{rpm}$ with $T_{0 A B}=850 \mathrm{~K}$.

A model for reduced mass flow rate and turbine efficiency has been built from the frozen rotor results at three fixed rotation speeds. The reduced mass flow rate is ap-

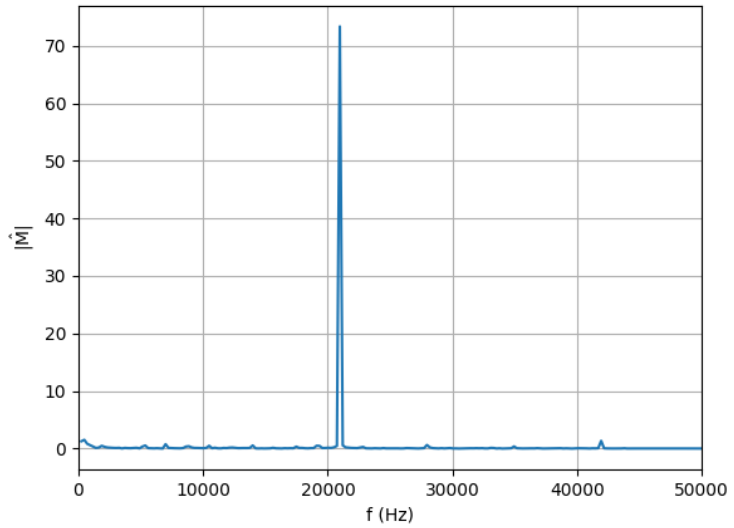

Fig. 12. Amplitudes of FFT of time dependent torque for TP1 regime.

proximated as

$$
\dot{m}_{\text {red }} \approx a_{0}+a_{1} \Pi+a_{2} n_{r e d}+a_{11} \Pi^{2}+a_{12} \Pi n_{\text {red }}+a_{22} n_{\text {red }}^{2},
$$

where the coefficients are $a_{0}=-3.902, a_{1}=8.192$, $a_{2}=-0.621 \times 10^{-3}, a_{11}=-2.213, a_{12}=0.473 \times 10^{-3}$, and $a_{22}=-0.105 \times 10^{-6}$. The figure 18 compares the reduced mass flow rate predictions given by the model (13) with experimental data and with the results of CFD simulations. One can see that the differences between the model predictions and the results are less than $2 \%$ with the exception of TP5 and TP9 regimes. In TP5 regime the unsteady simulation gives lower value of the mass flow rate whereas experimental data corresponds well to the model. Similarly in the TP9 the unsteady CFD predicts 


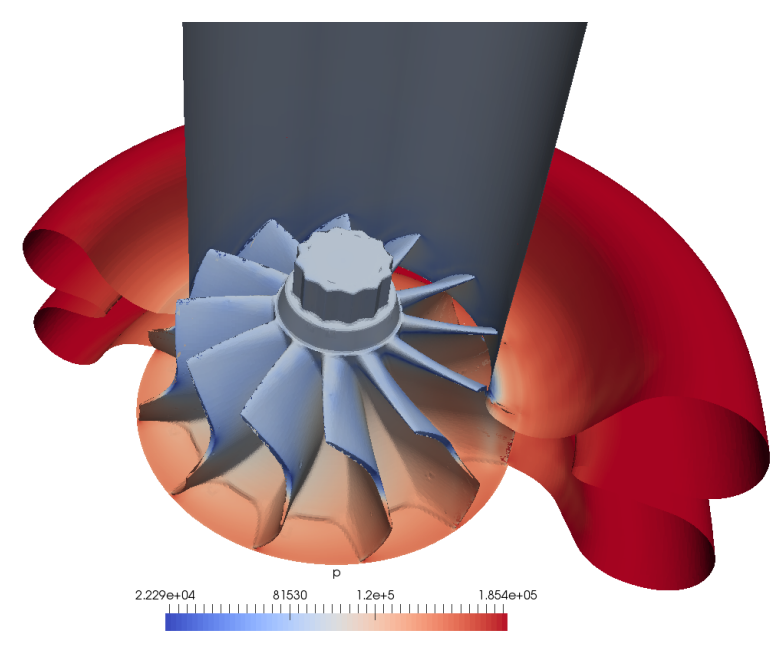

Fig. 13. Pressure distribution at solid walls in the twin scroll turbine, regime TP 1 .

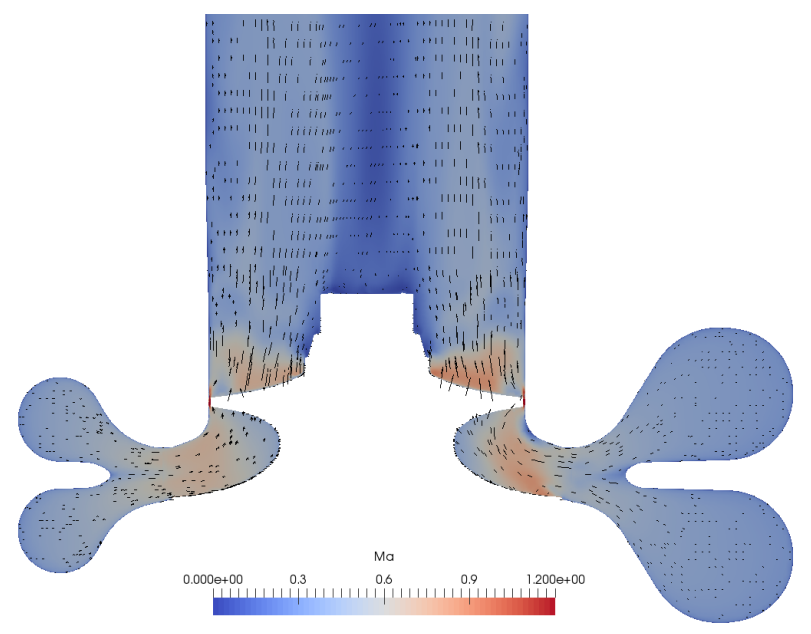

Fig. 14. Mach number in the cut through twin scroll turbine, regime TP 1 .

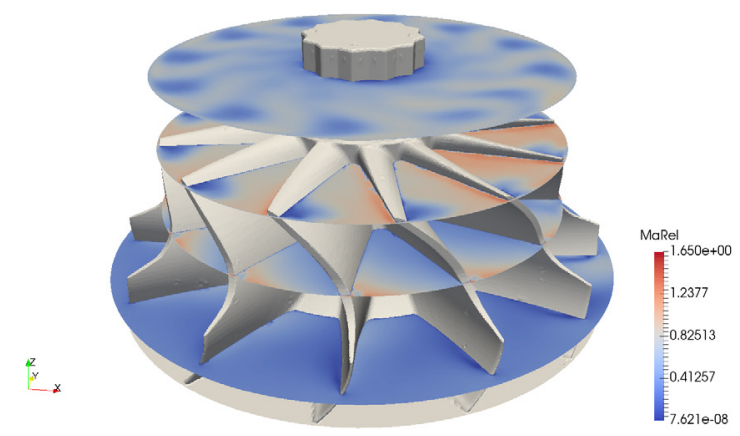

Fig. 15. Relative Mach number in cuts through rotor part of twin scroll turbine, regime TP 1.
Table 3. Expansion ratio $\Pi=p_{0 A B} / p_{\text {out }}$ and reduced rotation speed $n_{\text {red }}=R P M / \sqrt{T_{0 A B}}$ for twin scroll turbine analysis.

\begin{tabular}{|l|l|c|}
\hline Run & $\Pi(-)$ & $n_{\text {red }}(\mathrm{rpm} / \sqrt{K})$ \\
\hline \hline TP 1 & 2.246 & 3526 \\
\hline TP 2 & 2.242 & 3670 \\
\hline TP 3 & 2.283 & 2703 \\
\hline TP 4 & 1.627 & 2870 \\
\hline TP 5 & 2.213 & 4330 \\
\hline TP 6 & 1.665 & 2218 \\
\hline TP 7 & 1.667 & 2052 \\
\hline TP 8 & 1.261 & 2132 \\
\hline TP 9 & 1.595 & 3353 \\
\hline TP 10 & 1.268 & 1833 \\
\hline
\end{tabular}

Table 4. Comparison of reduced mass flow rate and turbine efficiency obtained with the experiment (columns "Exp"), by the steady state simulation (columns "Steady") and transient simulation (columns "Trans").

\begin{tabular}{|l|l|l|l|l|l|l|}
\hline \multirow{2}{*}{ Run } & \multicolumn{2}{|c|}{$\dot{m}_{\text {red }}\left(\mathrm{kg} \mathrm{s}^{-1} \sqrt{K} /\right.$ bar $)$} & \multicolumn{3}{|c|}{$\eta(\%)$} \\
\cline { 2 - 7 } & Exp & Steady & Trans & Exp & Steady & Trans \\
\hline \hline TP 1 & 3.370 & 3.411 & 3.365 & 64.6 & 65.3 & 65.3 \\
\hline TP 2 & 3.303 & 3.371 & 3.309 & 64.4 & 64.2 & 63.9 \\
\hline TP 3 & 3.613 & 3.570 & 3.540 & 60.8 & 61.4 & 61.7 \\
\hline TP 4 & 3.030 & 3.066 & 3.021 & 68.1 & 62.1 & 62.4 \\
\hline TP 5 & 3.092 & 3.112 & 2.839 & 52.4 & 54.6 & 45.7 \\
\hline TP 6 & 3.321 & 3.329 & 3.300 & 64.8 & 63.4 & 62.9 \\
\hline TP 7 & 3.373 & 3.371 & 3.352 & 61.0 & 61.8 & 61.7 \\
\hline TP 8 & 2.332 & 2.242 & 2.219 & 70.5 & 58.2 & 57.7 \\
\hline TP 9 & 2.815 & 2.665 & 2.487 & 61.1 & 47.4 & 45.0 \\
\hline TP 10 & 2.457 & 2.444 & 2.424 & 70.9 & 64.4 & 64.2 \\
\hline
\end{tabular}




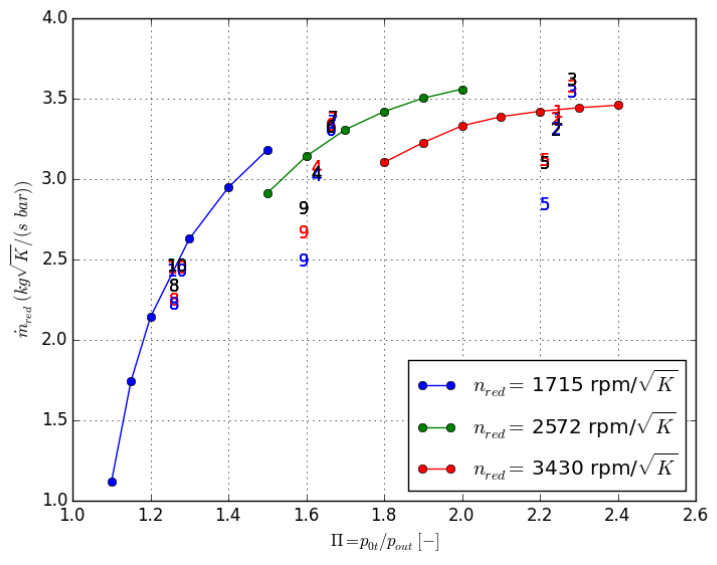

Fig. 16. Reduced mass flow rate for twin scroll turbine (experimental data depicted as black numbers, steady state simulation as red numbers, and transient simulation as blue numbers).

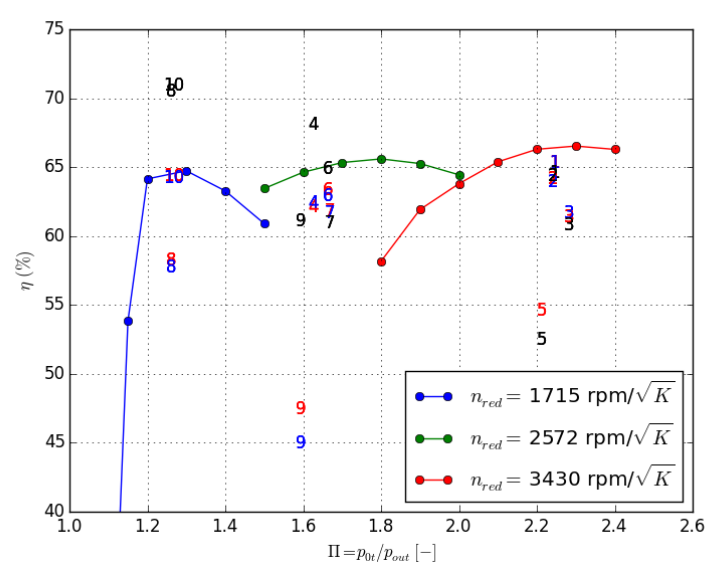

Fig. 17. Efficiency of twin scroll turbine (experimental data depicted as black numbers, steady state simulation as red numbers, and transient simulation as blue numbers).

lower mass flow rate whereas the experimental data gives a slightly higher value.

Similarly the efficiency can be approximated as

$$
\dot{\eta} \approx b_{0}+b_{1} \Pi+b_{2} n_{\text {red }}+b_{11} \Pi^{2}+b_{12} \Pi n_{\text {red }}+b_{22} n_{\text {red }}^{2},
$$

with $b_{0}=53.333, b_{1}=26.649, b_{2}=-11.255 \times 10^{-3}$, $b_{11}=-38.737, b_{12}=44.195 \times 10^{-3}$, and $b_{22}=$ $-12.994 \times 10^{-6}$.

The figure 19 compares the predicted efficiency given by the model (14) with the efficiency evaluated from the experimental data and from the CFD simulations. One can see that most of the data falls into $5 \%$ region, however the experimental data give slightly higher efficiencies in regimes TP4, TP8, TP9, and TP10 whereas the unsteady simulations predict lower efficiency in regimes TP5 and TP9.

Note that the regimes TP5 and TP9 where the models for $\dot{m}_{r e d}$ and $\eta$ differ from the experimental data and

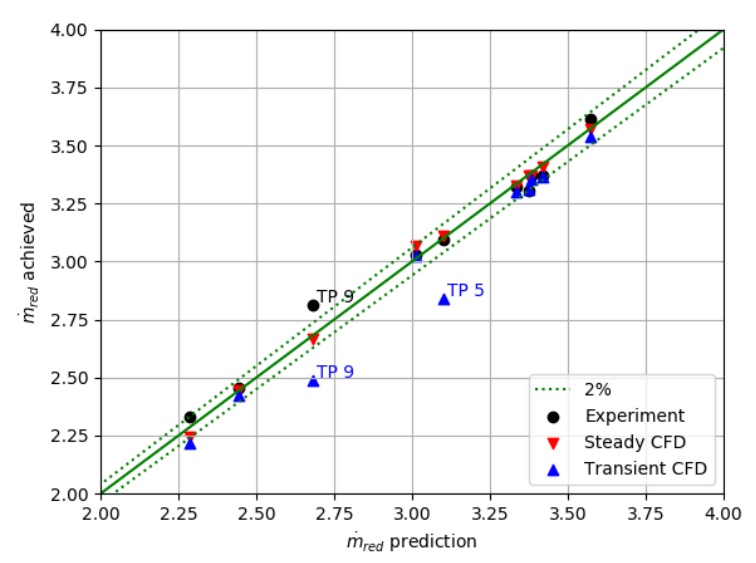

Fig. 18. Comparison of the mass flow rate model (13) with experimental and CFD results.

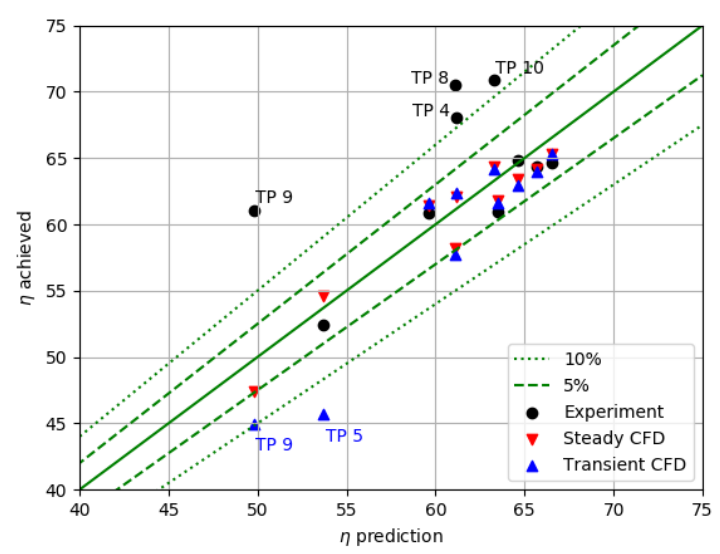

Fig. 19. Comparison of the efficiency model (14) with experimental and CFD results.

unsteady simulations are characterized by lower inlet temperature resulting in higher Mach numbers in the turbine when comparing to other regimes.

\section{Conclusion}

The LU-SGS solver has been extended for turbomachinery flows using stationary frozen-rotor and unsteady sliding mesh approaches. The solver has been validated using the ERCOFTAC centrifugal pump comparing the results both with experimental data and with the pressure based solver from the OpenFOAM package. Finally the solver has been used for the analysis of the flows through a twinscroll turbine. The comparison of simulation results using both approaches with the experimental data shows that the mass flow rate is predicted within $2 \%$ error by both computational approaches and that a reasonable agreement between predicted and measured efficiency can be achieved. Although the frozen rotor approach completely disregards the unsteady character of turbomachinery flow, the current study shows that it can be viable alternative to much 
more computationally demanding sliding mesh method for flows in radial turbines.

Acknowledgements. Authors acknowledge support from the EU Operational Programme Research, Development and Education, and from the Center of Advanced Aerospace Technology (CZ.02.1.01/0.0/0.0/16_019/0000826), Faculty of Mechanical Engineering, Czech Technical University in Prague and support by TACR, project no. TE01020020.

\section{References}

1. M. Ubaldi, P. Zunino, G. Barigozzi, A. Cattanei, J. Turbomach. 118, 41 (1996)

2. S.V. Patankar, D.B. Spalding, Int. J. Heat Mass Transf. 15, 1787 (1972)

3. R. Issa, J. Comput. Phys. 62, 40 (1986)

4. J. Benajes, J. Galindo, P. Fajardo, R. Navarro, J. Appl. Fluid Mech. 7, 673 (2014)

5. L. Mangani, M. Darwish, F. Moukalled, in Proceedings of ASME TURBO EXPO 2014 (ASME, Düsseldorf, 2014), p. 13
6. J. Fürst, in EPJ Web of Conferences (2017), Vol. 143, p. 02027

7. A. Kurganov, E. Tadmor, J. Comput. Phys. 160, 241 (2000)

8. M.S. Liou, J. Comput. Phys. 214, 137 (2006)

9. E.F. Toro, in Riemann Solvers and Numerical Methods for Fluid Dynamics (Springer Berlin Heidelberg, Berlin, Heidelberg, 1997), pp. 293-311

10. J. Blazek, Computational fluid dynamics : principles and applications (Butterworth-Heinemann, 2015)

11. C. Shen, X.1. Xia, Y.z. Wang, F. Yu, Z.w. Jiao, Adv. Eng. Softw. 91, 80 (2016)

12. J. Fürst, in 12th OpenFOAM workshop (Exeter, 2017)

13. O. Petit, M. Page, M. Beaudoin, H. Nilsson, in IAHR International Meeting of the Workgroup on Cavitation and Dynamic Problems in Hydraulic Machinery and Systems (2009), pp. 523-532

14. O. Petit, H. Nilsson, Int. J. Rot. Mach. 2013, 1 (2013)

15. T. Barth, D. Jespersen, in 27th Aerospace Sciences Meeting (AIAA, Reston, Virigina, 1989)

16. F.R. Menter, AIAA Journal 32, 1598 (1994) 